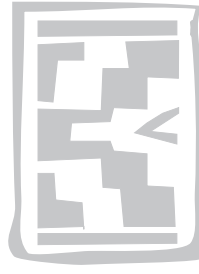

\title{
Molecular monitoring of African swine fever virus using surveys targeted at adult Ornithodoros ticks: a re-evaluation of Mkuze Game Reserve, South Africa
}

\author{
L.F. ARNOT'1, 2 , J.T. DU TOIT ${ }^{1,3}$ and A.D.S. BASTOS ${ }^{1,4 *}$
}

\begin{abstract}
ARNOT, L.F., DU TOIT, J.T. \& BASTOS, A.D.S. 2009. Molecular monitoring of African swine fever virus using surveys targeted at adult Ornithodoros ticks: a re-evaluation of Mkuze Game Reserve, South Africa. Onderstepoort Journal of Veterinary Research, 76:385-392

The Mkuze Game Reserve (MGR), in north-eastern KwaZulu-Natal Province, South Africa is an African swine fever virus (ASF) controlled area. In a survey conducted in 1978, ASF prevalence in warthogs and Ornithodoros ticks in MGR was determined to be $2 \%$ and $0.06 \%$, respectively. These values, acknowledged as being unusually low compared to other East and southern African ASFpositive sylvatic-cycle host populations, have not been assessed since. The availability of a sensitive PCR-based virus detection method, developed specifically for the sylvatic tampan host, prompted a re-evaluation of ASF virus (ASFV) prevalence in MGR ticks. Of the 98 warthog burrows inspected for Ornithodoros presence, $59(60.2 \%)$ were found to contain tampans and tick sampling was significantly male-biased. Whilst gender sampling-bias is not unusual, the $27 \%$ increase in infestation rate of warthog burrows since the 1978 survey is noteworthy as it anticipates a concomitant increase in ASFV prevalence, particularly in light of the high proportion (75\%) of adult ticks sampled. However, despite DNA integrity being confirmed by internal control amplification of the host 16S gene, PCR screening failed to detect ASFV. These results suggest that ASFV has either disappeared from MGR or if present, is localized, occurring at exceptionally low levels. Further extensive surveys are required to establish the ASFV status of sylvatic hosts in this controlled area.
\end{abstract}

Keywords: African swine fever virus, Mkuze Game Reserve, Ornithodoros porcinus, PCR, warthog

\section{INTRODUCTION}

African swine fever (ASF) is one of the most infectious diseases of domestic pigs, with mortality approaching $100 \%$ in commercially farmed animals

* Author to whom correspondence is to be directed. E-mail: adbastos@zoology.up.ac.za

1 Mammal Research Institute, Department of Zoology and Entomology, University of Pretoria, Pretoria, 0002 South Africa

2 Current address: Department of Paraclinical Sciences, Faculty of Veterinary Science, University of Pretoria, Private Bag X04, Onderstepoort, 0110 South Africa

3 Current address: Department of Wildland Resources, Utah State University, Logan, UT 84322-5230, USA

4 ARC-Onderstepoort Veterinary Institute, Private Bag X05, Onderstepoort, 0110 South Africa

Accepted for publication 13 April 2009-Editor
(Plowright, Thomson \& Neser 1994). ASF is a controlled animal disease in South Africa (under Animal Diseases Act 35 of 1984) that has significant epizootic potential (Thomson 1999). Slaughter and total area quarantine are the only known methods of control as there is presently no vaccine available to combat the disease (Penrith, Thomson \& Bastos 2004). ASF outbreaks have severely affected the pig industry in southern, East and West Africa in recent years (Penrith et al. 2004; Penrith, Lopes Pereira, Lopes da Silva, Quembo, Nhamusso \& Banze 2007) decimating pig stocks on both subsistence and commercial farms.

The natural arthropod host, a long-lived, eyeless, warthog-burrow-dwelling argasid tick of the genus 
Ornithodoros (Kleiboeker \& Scoles 2001), is the most important maintenance host and vector of the ASF virus (ASFV) in Africa (Plowright, Parker \& Peirce 1969; Thomson 1985). The distribution of ASF in southern Africa is determined largely by the distribution of this sylvatic cycle soft tick and its preferred vertebrate host, the common warthog Phacochoerus africanus (Thomson 1985). In the invertebrate tick host, the virus can be transmitted sexually, trans-ovarially and trans-stadially (reviewed by Kleiboeker \& Scoles 2001). Infection rates increase with increasing size and age of the tick, and females display higher infection rates than males, due most likely to directional sexual transmission of the virus from males to females (Plowright 1977). In the absence of an infectious vertebrate blood meal, the virus can be maintained for periods of up to 15 months in the sylvatic Ornithodoros tick (Plowright, Perry, Pierce \& Parker 1970) and possibly even indefinitely (Plowright 1977). Whilst the virus can persist for prolonged periods in infected ticks, which are considered to be the hosts of primary importance in ASF epidemiology, warthogs play an important role in amplifying the virus in tick colonies. This ostensibly occurs at those times when virus levels in viraemic neonate warthogs surpass the threshold level required to infect ticks (Thomson 1985; Plowright et al. 1994). As warthogs are seasonal breeders in southern Africa, the proposed viral transmission between the vertebrate and invertebrate hosts is cyclical, coinciding with the warthog farrowing season that runs from November to December (Cumming 2005). ASFV seroprevalence in large ASF-infected warthog populations is generally high, ranging from $50-100 \%$, while the presence of virus in extensively surveyed East African tick populations is substantially lower, ranging from 0.26 to $5.03 \%$ in adult ticks (Plowright 1977).

A study was undertaken in 1978 to assess the ASF status in warthog and Ornithodoros sylvatic cycle hosts in Mkuze Game Reserve (MGR), South Africa (Thomson, Gainaru, Lewis, Biggs, Nevill, Van der Pypekamp, Gerber, Esterhuysen, Bengis, Bezuidenhout \& Condy 1983; Thomson 1985). In this study, the ASFV infection rate for 5018 ticks was determined by inoculating pig leucocyte cultures with pooled ground tick suspensions ( $3-5$ adult females, 6-8 adult males and >10 nymphs; Thomson et al. 1983). Following blind passage, three haemadsorption-(HAd)-negative isolates, of reduced virulence for domestic pigs, were obtained, corresponding to a $0.06 \%$ infection rate. This is 23 -fold lower than the ASFV infection rate in Ornithodoros in the Kruger National Park (KNP), South Africa (Thomson et al.
1983). Similarly, ASFV seroprevalence in warthogs in MGR was unusually low (2\%) on immuno-electroosmophoresis (IEOP) compared to the $94 \%$ on IEOP for KNP, with ELISA seroprevalence estimates being $6 \%$ and $85 \%$, respectively. The MGR prevalence levels were the lowest documented for the six ASF-positive southern African localities evaluated (Thomson et al. 1983), and remain the lowest reported thus far for an ASF-positive region in which substantial numbers of both sylvatic hosts co-occur. The exceptionally low ASFV prevalence in MGR, availability of a sensitive PCR-based method for determining infection rates in ticks (Bastos, Arnot, Jacquier \& Maree, 2009), lack of large-scale surveys since 1978 and the recent incorporation of MGR into the Greater St Lucia Wetland Park, prompted a re-evaluation of the ASFV status in the sylvatic host that is of primary importance in ASF epidemiology, the warthog-burrow-dwelling Ornithodoros tampan.

\section{MATERIALS AND METHODS}

\section{Study area and warthog census data}

The MGR is a 37000 ha conservation area situated in north-eastern KwaZulu-Natal (KZN) Province, South Africa and constitutes the north-western spur of the Greater St Lucia Wetland Park. The park is flanked to the north and south by the high-prevalence ASF and zero-prevalence warthog populations of the KNP and Hluhluwe-iMfolozi Park (HiP), respectively. In 1984, the number of warthog estimated to be present in the northern (main) part of the reserve (Fig. 1) was 630, while during the 2002 game census conducted by the KZN Nature Conservation Service in the same part of the reserve, the warthog population was estimated to be in the vicinity of 1000 individuals (Craig Mulqueeny, personal communication 2002).

\section{Tick collection}

A total of 98 warthog burrows throughout the reserve were sampled for tampans (Fig. 1). The exact position of each burrow was recorded using a hand held GPS unit. Loose soil was removed from the burrows with a spade and sieved over a white plastic sheet, using a stainless-steel Labotec test sieve, with apertures of $3350 \mu \mathrm{m}$. The plastic sheet facilitated the detection of small ticks that passed through the sieve.

Ticks were transported to the University of Pretoria in sealed containers, under a permit issued by the Department of Agricultural Technical Services, and stored at $-70^{\circ} \mathrm{C}$ until they were required for anal- 


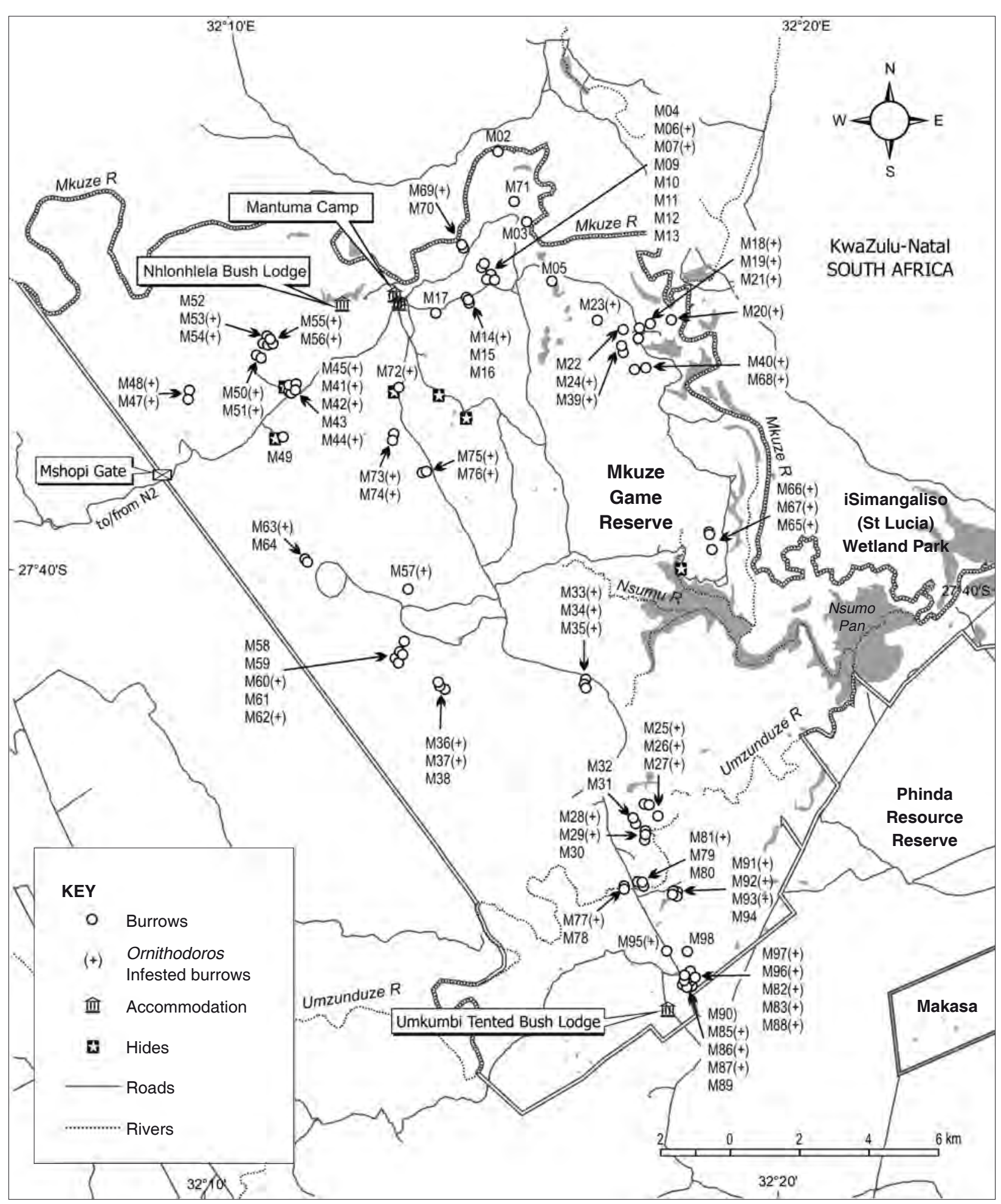

FIG. 1 Map of Mkuze Game Reserve depicting the distribution of the 98 warthog burrows (M1-M98) investigated for the presence of Ornithodoros

ysis. The species designation Ornithodoros porcinus for ticks collected is used here in accordance with the results of a molecular phylogenetic analysis of geographical diversity in the tick host of ASFV
(Bastos et al. 2009). This analysis confirmed the monophyly of $O$. porcinus but revealed a lack of support for the O.p. porcinus and O.p. domesticus sub-species designations. The ventral and dorsal 
surfaces of all ticks were photographed and they were visually sexed using the guidelines of Walton (1962), and classed as male, female or nymphs. A two sample t-test was used to test for any difference in the overall occurrence of male and female ticks across all samples collected in MGR, using GENSTAT version 4.2 (Lane \& Payne 1996).

\section{Molecular analyses}

DNA was extracted using a silica-guanidine thiocyanate technique from $10 \% \mathrm{~W} / \mathrm{V}$ ground tick suspensions, prepared as described previously (Bastos et al. 2009). ASFV genome presence was evaluated using a duplex PCR approach that, in addition to being confirmatory for the virus, is also informative with respect to host taxonomy and ASFV genotype (Bastos et al. 2009). False negative results, due either to a failed PCR or a failed DNA extraction, are precluded through amplification of the tick mitochondrial 16S gene, which acts as an internal control. Positive and negative controls were always included to ensure reaction efficiency and freedom from contamination of the reagents used.

To determine the genetic relatedness of the three MGR 1978 viruses to each other and to previously characterised, regional viruses, nucleotide sequences corresponding to the C-terminal end of the $p 72$ gene were generated as previously described (Bastos, Penrith, Cruciere, Edrich, Hutchings, Roger, Couacy-Hymann \& Thomson 2003). In addition, three South African tampan isolates sampled between 1979 and 1987, were characterised. Sequences generated by a manual sequencing approach were submitted to Genbank (www.ncbi.nlm.nih.gov) under accession numbers FJ455835 to FJ455840, and were complemented with homologous reference sequence data available for each of the 14 southern African genotypes described to date (Bastos et al. 2003; Boshoff, Bastos, Gerber \& Vosloo 2007). A molecular phylogeny was inferred in MEGA4 (Tamura, Dudley, Nei \& Kumar 2007) using the neighbour-joining algorithm with nodal support being assessed by 10000 bootstrap replications.

\section{RESULTS}

\section{Burrow infestation rate and sex ratios of adult ticks}

Tampans were collected from 59 of the 98 burrows inspected, which corresponds to a $60.2 \%$ tick infestation rate. Of the 348 ticks collected, $88(25 \%)$ were nymphs and 161 of the 260 adult ticks (62\%) were males. This 62:38 male:female bias was statistically significant $(P<0.01$; two-sample t-test).

\section{Screening for ASFV genome presence}

All 348 ticks were screened for the presence of ASFV using the sylvatic tick duplex PCR (Bastos et al. 2009). None of these 348 ticks amplified the expected 478 bp ASFV p72 gene target. However, with the exception of the negative controls, all reactions amplified the expected 313 bp 165 host mitochondrial gene target of the internal control, thereby confirming template DNA and reaction integrity and precluding the possibility of false negative results.

\section{Retrospective genetic analysis of the $\mathbf{1 9 7 9}$ Mkuze tick isolates}

Nucleotide sequencing of the C-terminal end of the p72 gene revealed that the three MGR tick viruses isolated in 1978 were identical to each other and also to two viruses that caused outbreaks of ASF in domestic pigs in 1973 and 1975 in South Africa (Fig. 2). Phylogenetic analysis of the homologous 401 nucleotide (nt) dataset identified the MGR viruses as belonging to genotype $\mathrm{XX}$, whilst three additional South African tampan viruses clustered within southern African, domestic pig genotypes III and XXII. None of the tick viruses were unique across the gene region sequenced, with each being identical to a domestic pig virus (Fig. 2).

\section{DISCUSSION}

The two notable differences between the 1978 and 2002 surveys in the MGR with respect to the sylvatic hosts of ASFV, relate to changes in burrow infestation as well as warthog numbers. O. porcinus were found in 59 out of the 98 burrows surveyed (60\% infestation rate) in 2002, whilst Thomson (1985) reported that ticks were retrieved from 13 out of the 40 burrows (33\%) surveyed in 1978 in MGR. These results indicate that tick populations were present in $27 \%$ more burrows in 2002 than in 1978, probably due to the estimated $59 \%$ increase in warthog numbers from 1984 to 2002. The marked increase in warthog numbers, together with the observation that $10.8 \%$ and $47 \%$ of warthogs sampled outside their burrows in Namibia and in South Africa, respectively, were infested with Ornithodoros nymphs (Horak, Biggs, Hanssen \& Hanssen, 1983; Horak, Boomker, De Vos \& Potgieter, 1988), lends credence to the proposed role played by warthogs in ASF epidemiology, as disseminators of the inver- 


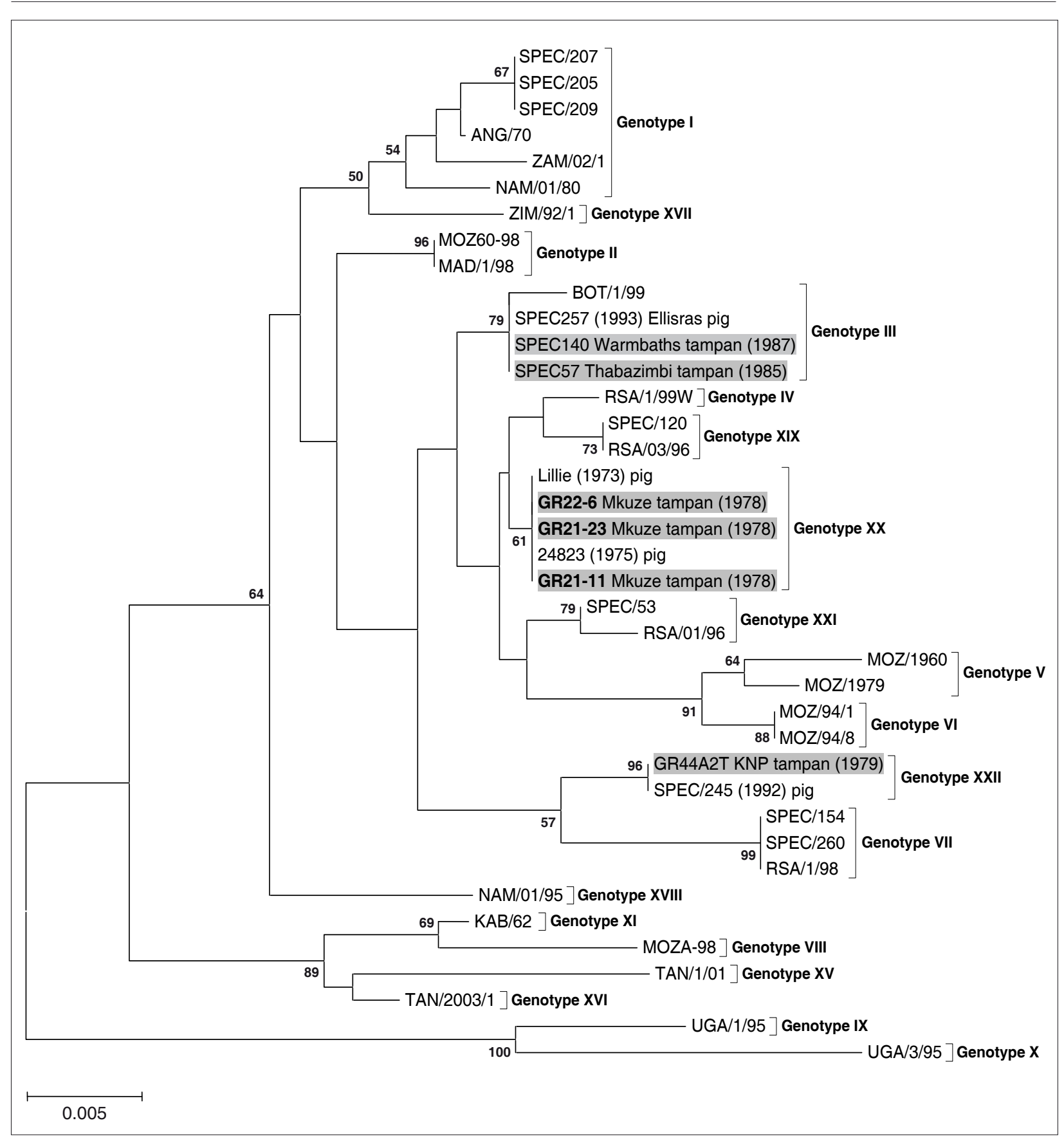

FIG. 2 Genetic relatedness of the three Mkuze Game Reserve tampan viruses isolated in 1978 (indicated in bold with grey shading) and three additional South African tampan viruses (denoted by grey shading alone), based on C-terminal p72 gene sequence analysis

Nodal support $\geq 50 \%$ from 10000 bootstrap replicates is indicated next to each node and the previously defined genotypes recovered following neighbor-joining analysis of the homologous $401 \mathrm{nt}$ dataset, are shown to the right of the relevant lineage

tebrate tick host (Thomson 1985). An increase in ASFV prevalence could thus be anticipated in MGR because of the increase in warthog numbers and concomitant increase in burrow infestation rates and resulting tick-warthog contacts. This was not, however, the case. Instead, none of the 348 ticks screened using a molecular approach developed specifically for ASFV screening of Ornithodoros porcinus (Bastos et al. 2009) harboured the virus.

Analysis of the C-terminal $p 72$ gene region revealed that the three MGR viruses isolated from pooled 
ticks sampled in 1978 were identical to each other and to two historical, genotype XX domestic pig strains, recovered from ASF outbreaks in South Africa, in 1973 and 1975 (Fig. 2). These MGR viruses were, however, distinct from the three other South African tampan isolates, with the Kruger National Park (KNP) tampan virus clustering within genotype XXII and the Thabazimbi and Warmbaths (Bela Bela) tick viruses being assigned to genotype III. The two domestic pig viruses, Lillie and 24823, shown to be identical to the MGR tick viruses, were also HAd-negative, with isolate 24823 having reduced virulence in domestic pigs (Pini 1977). These results are consistent with the observations of Thomson and co-workers (1983) that the three Mkuze viruses were unusual for their non-haemadsorbing characteristic and reduced virulence in domestic pigs. Other atypical features of the ASF-status of the MGR sylvatic hosts are the unusually low seroprevalence of the vertebrate (warthog) host (2\% and $6 \%$ on IEOP and ELISA, respectively). In particular, it was noted that only one of three warthog sub-yearlings from the same sounder, was found to be seropositive to ASF (Thomson et al. 1983). This unexpected result indicates that the specificity/ accuracy of ASF serological tests in warthogs is possibly sub-optimal and underscores the need to investigate and validate tests specifically for sylvatic vertebrate hosts (Jori \& Bastos 2009).

Transformation of the ASFV incidence by sex and size class of Ornithodoros ticks sampled at three southern African localities (Table 4 of Thomson et al. 1983) revealed that at all localities, more adult males than females were recovered, with the average male:female ratio being 61:39. The 62:38 ratio recorded in the 2002 MGR survey is therefore consistent with these previous observations of male bias. However, differences in the size classes were pronounced with substantially more nymphs than adults being collected in earlier surveys (Pini 1977; Thomson et al. 1983). With the exception of KNP, 6.7 more nymphs than adults (13\% adults: $87 \%$ nymphs) on average, were collected from tick populations sampled in the 1970s by Thomson and coworkers (1983). In contrast, sampling was skewed towards adults (75:25 adults:nymphs) in the 2002 survey due most likely to differences in the aperture size of the sieves used in the 1978 versus 2002 survey. This higher proportion of adults to nymphs in the 2002 sample is relevant as a nine-fold higher ASFV infection rate was observed in adult ticks versus nymphs ( $2.5 \%$ versus $0.27 \%$ ), on average, in three southern Africa tick populations based on data provided in Table 4 of the Thomson et al. (1983) study.

Whereas it may be tempting to attribute the negative results obtained from the 2002 survey to inadequate sampling, as just 348 ticks were screened compared to 5018 in the 1978 survey, a number of important differences should be borne in mind. First, warthog numbers and burrow infestation rates were $59 \%$ and $27 \%$ higher during the 2002 survey. Second, an almost six-fold higher proportion of adult ticks were collected in 2002 compared to 1978 . Third, PCR was found to be 1.4 times more sensitive than ASFV isolation from Ornithodoros ticks in Europe following first-round amplification alone (Basto, Portugal, Nix, Cartaxeiro, Boinas, Dixon, Leitão \& Martins 2006). This result is in keeping with those from studies on other viruses which found that PCR was between 1.4 and 1.5 times more sensitive than virus isolation (Risatti, Holinka, Lu, Kutish, Callahan, Nelson, Brea Tió \& Borca 2005; Wang, O'Keefe, Orr, Loth, Banks, Wakeley, West, Card, Ibata, Van Maanen, Thoren, Isaksson \& Kerkhofs 2008). Therefore, even if the increase in warthog numbers and burrow infestations is discounted and only differential, adult tick infection rate (nine-fold higher in adults versus nymphs) and the 1.4-fold higher sensitivity afforded by PCR screening are considered, it can be determined that in spite of the smaller sample size, a five-fold increase (from 0.06 to $0.30 \%$ ) in ASFV prevalence in the 2002 MGR tick sample was a reasonable expectation. The negative result, despite more burrows and proportionally more adults being screened using a PCRbased approach that precludes false negatives, makes it highly improbable that any ASFV-positive tick colonies went undetected in the 2002 survey. This then begs the question as to whether ASFV still exists within the MGR. If the virus still persists then it must be extremely localized and restricted to burrows that were not surveyed in our study.

As ASF is a controlled disease of economic significance, it has major implications for the development of pig industries in northern KZN and also impacts on trade in indigenous swine. Any change in ASFV infection of sylvatic hosts in MGR would require reassessment of the continued inclusion of MGR within the ASF control area. This PCR-based pilot study has highlighted the possible disappearance of the virus from MGR and the value of performing adult Ornithodoros tick targeted molecular surveys. It has also indentified a need for further, more extensive studies to establish beyond doubt whether ASFV still persists within the greater MGR area. 


\section{CONCLUSION}

Ninety-eight burrows examined for the presence of $O$. porcinus in MGR yielded 348 O. porcinus ticks in 59 burrows. Despite a $27 \%$ increase in burrow infestation and $59 \%$ increase in warthog density since 1978, a nearly six-fold increase in proportional sampling of adult ticks versus nymphs, and employment of a sensitive PCR screening method, no evidence of ASFV genome presence could be found. It is therefore possible that ASFV no longer exists within MGR and that if it does, that the ASF infection rate is extremely low and restricted to a small number of warthog burrows. We propose that molecular screening of adult ticks is reliable and cost-effective for evaluating ASFV control area status and for conducting ASFV surveillance in areas of particular concern.

\section{ACKNOWLEDGEMENTS}

We are grateful to Warren McCall for field assistance, Stephan Swanepoel for providing virus isolation information and Ingrid Booysen for preparing the Mkuze map. Financial support for the Mkuze tick survey was provided through an RDP and Thuthuka grant awarded to ADSB by the University of Pretoria and the National Research Foundation of South Africa.

\section{REFERENCES}

BASTO, A.P., PORTUGAL, R.S., NIX, R.J., CARTAXEIRO, C., BOINAS, F., DIXON, L.K., LEITÃO, A. \& MARTINS, C. 2006. Development of a nested PCR and its internal control for the detection of African swine fever virus (ASFV) in Ornithodoros erraticus. Archives of Virology, 151:819-826.

BASTOS, A.D.S., PENRITH, M-L., CRUCIERE, C., EDRICH, J., HUTCHINGS, G., ROGER, F., COUACY-HYMANN, E. \& THOMSON, G.R. 2003. Genotyping field strains of African swine fever virus by partial $p 72$ gene characterization. Archives of Virology, 148:693-706.

BASTOS, A., ARNOT, L., JACQUIER, M. \& MAREE, S. 2009. A host-species informative internal control for molecular assessment of African swine fever virus infection rates in the African sylvatic cycle Ornithodoros vector. Medical and Veterinary Entomology, 23:399-409.

BOSHOFF, C.I., BASTOS, A.D.S., GERBER, L.J. \& VOSLOO, W. 2007. Genetic characterisation of African swine fever viruses from outbreaks in southern Africa (1973-1999). Veterinary Microbiology, 121:45-55.

CUMMING, D.H.M. 2005. Superorder Cetartiodactyla, Order Suiformes, in The mammals of the southern African subregion, edited by J. Skinner \& C.T. Chimimba. Cambridge University Press: Cambridge.

HORAK, I.G., BIGGS, H.C., HANSSEN, T.S. \& HANSSEN, R.E. 1983. The prevalence of helminth and arthropod parasites of warthog, Phacochoerus aethiopicus, in South West Africa/ Namibia. Onderstepoort Journal of Veterinary Research, 50: 145-148.

HORAK, I.B., BOOMKER, J., DE VOS, V. \& POTGIETER, F.T. 1988. Parasites of domestic and wild animals in South Africa. XXIII. Helminth and arthropod parasites of warthog, Phacochoerus aethiopicus in the eastern Transvaal Lowveld. Onderstepoort Journal of Veterinary Research, 55:145-152.

JORI, F. \& BASTOS, A.D.S. 2009. Role of wild suids in the epidemiology of African swine fever. Ecohealth, 6:296-310.

KLEIBOEKER, S.B. \& SCOLES, G.A. 2001. Pathogenesis of African swine fever virus in Ornithodoros ticks. Animal Health Research Reviews, 2:121-128.

LANE, P.W. \& PAYNE, R.W. 1996. GENSTAT for windows. London: The numerical Algorithms Group.

PENRITH, M-L., THOMSON, G.R. \& BASTOS, A.D.S. 2004. African swine fever, in Infectious diseases of livestock, $2^{\text {nd }}$ edition, edited by J.A.W. Coetzer \& R.C. Tustin. Cape Town: Oxford University Press.

PENRITH, M-L., LOPES PEREIRA, C., LOPES DA SILVA, M.M., QUEMBO, C., NHAMUSSO, A. \& BANZE, J. 2007. African swine fever in Mozambique: review, risk factors and considerations for control. Onderstepoort Journal of Veterinary Research, 74:149-160.

PINI, A. 1977. Strains of African swine fever virus isolated from domestic pigs and from the tick Ornithodoros moubata in South Africa. D.V.Sc. thesis, University of Pretoria.

PLOWRIGHT, W., PARKER, J. \& PEIRCE, M.A. 1969. African swine fever virus in ticks (Ornithodoros moubata, Murray) collected from animal burrows in Tanzania. Nature, 221: 1071-1073.

PLOWRIGHT, W., PERRY, C.T., PIERCE, M.A. \& PARKER, J. 1970. Experimental infection of the argasid tick Ornithodoros moubata porcinus with African swine fever virus. Archiv für gesamte Virusforschung, 31:33-50.

PLOWRIGHT, W. 1977. Vector transmission of African swine fever virus. Hog cholera/classical swine fever and African swine fever. Commission of the European Communities.

PLOWRIGHT, W., THOMSON, G.R. \& NESER, J.A. 1994. African swine fever, in Infectious diseases of livestock with special reference to southern Africa, edited by J.A.W. Coetzer, G.R. Thomson \& R.C. Tustin. Cape Town: Oxford University Press.

RISATTI, G., HOLINKA, L., LU, Z., KUTISH, G., CALLAHAN, J.D., NELSON, W.M., BREA TIÓ, E. \& BORCA, M.V. 2005. Diagnostic evaluation of a real-time reverse transcriptase PCR assay for detection of Classical swine fever virus. Journal of Clinical Microbiology, 43:468-471.

TAMURA, K., DUDLEY, J., NEI, M. \& KUMAR, S. 2007. MEGA4: Molecular Evolutionary Genetics Analysis (MEGA), version 4.0. Molecular Biology and Evolution, 24:1596-1599.

THOMSON, G., GAINARU, M.D., LEWIS, A., BIGGS, H., NEVILL, E., VAN DER PYPEKAMP, H., GERBER, L., ESTERHUYSEN, J., BENGIS, R., BEZUIDENHOUT, D. \& CONDY, J. 1983. The relationship between African swine fever virus, the warthog and Ornithodoros species in southern Africa, in African swine fever, edited by P.J. Wilkinson. Commission of the European Communities.

THOMSON, G.R. 1985. The epidemiology of African swine fever: The role of free living hosts in Africa. Onderstepoort Journal of Veterinary Research, 52:201-209. 
Molecular monitoring of African swine fever virus: re-evaluation of Mkuze Game Reserve, South Africa

THOMSON, G.R. 1999. Alternatives for controlling animal diseases resulting from interaction between livestock and wildlife in southern Africa. South African Journal of Science, 95: 71-76.

WALTON, G.A. 1962. The Ornithodoros moubata superspecies problem in relation to human relapsing fever epidemiology. Zoological Society of London, 6:83-156.
WANG, J. O'KEEFE, J., ORR, D., LOTH, L., BANKS, M., WAKELEY, P., WEST, D., CARD, R., IBATA, G., VAN MAANEN, K., THOREN, P., ISAKSSON, M. \& KERKHOFS, P. 2008. An international inter-laboratory ring trial to evaluate a real-time PCR assay for the detection of bovine herpesvirus 1 in extended bovine semen. Veterinary Microbiology, 126:11-19. 